\title{
D-(+)-Galactose-Conjugated Single-Walled Carbon Nanotubes as New Chemical Probes for Electrochemical Biosensors for the Cancer Marker Galectin-3
}

Young Kum Park ${ }^{1}$, Bayarmaa Bold ${ }^{1}$, Woo Kyung Lee ${ }^{1}$, Min Hyon Jeon ${ }^{1}$, Kay Hyeok An ${ }^{2}$, Seung Yol Jeong ${ }^{3}$ and Young Key Shim ${ }^{1, *}$

1 Department of Nano System Engineering, Inje University, Gimhae, 612-749, Korea;

E-Mails: immergreen@dreamwiz.com (Y.K.P.); bayarma_sbmt@yahoo.com (B.B.); wlee@inje.ac.kr (W.K.L.); mjeon@inje.ac.kr (M.H.J.)

2 Carbon Valley R \& D Division, Jeonju Institute of Machinery and Carbon Composites, Jeonju, 561-844, Korea; E-Mail: khan@jmc.re.kr

3 Nano Carbon Materials Research Group, Korea Electrotechnology Research Institute, Changwon, 641-120, Korea; E-Mail: syjeong@ keri.re.kr

* Author to whom correspondence should be addressed; E-Mail: ykshim@inje.ac.kr; Tel.: +82-055-320-3871; Fax: +82-055-321-7034.

Received: 6 April 2011; in revised form: 22 April 2011 / Accepted: 29 April 2011 /

Published: 5 May 2011

\begin{abstract}
D-(+)-Galactose-conjugated single-walled carbon nanotubes (SWCNTs) were synthesized for use as biosensors to detect the cancer marker galectin-3. To investigate the binding of galectin-3 to the D-(+)-galactose-conjugated SWCNTs, an electrochemical biosensor was fabricated by using molybdenum electrodes. The binding affinities of the conjugated SWCNTs to galectin-3 were quantified using electrochemical sensitivity measurements based on the differences in resistance together with typical I-V characterization. The electrochemical sensitivity measurements of the D-(+)-galactose-conjugated SWCNTs differed significantly between the samples with and without galectin-3. This indicates that D-(+)-galactose-conjugated SWCNTs are potentially useful electrochemical biosensors for the detection of cancer marker galectin-3.
\end{abstract}

Keywords: biosensor; cancer marker; galectin-3; galactose; single-walled carbon nanotubes 


\section{Introduction}

Galectins, a family of $\beta$-galactoside-binding proteins, have been strongly implicated in cancer and may be useful targets for the development of new cancer detection methods [1,2]. So far, 14 mammalian galectins have been identified, all of which contain a conserved carbohydrate recognition binding domain (CRD) of approximately 130 amino acids. Of the galectins, galectin-3 is the most studied member of the galectin family. High levels of circulating galectin-3 are correlated with an increased potential for malignancy in several types of cancer [3]. Currently, a convenient and economical method is not available for detecting galectins in tissue samples, although antibody based methods such as enzyme-linked immunosorbent assays (ELISA) and Western blotting are in use. A method based on a chemical probe shows potential as an alternative technique [4].

The development of advanced biosensor devices has emerged as the most promising short-term application of carbon nanotubes (CNTs) in biology and medicine. CNTs offer new opportunities for rapid, sensitive, and label-free detection of biological agents, and biofunctionalization confers selectivity of detection on the CNTs [5]. The use of single-walled CNTs (SWCNTs) in biosensors has been reported [6-18]. Currently, research on CNT-based biosensors is focused on exploiting the development of CNT electrodes for the electrochemical detection of biological agents, such as glucose [19], immunoglobulin G (IgG) [20], immunoglobulin E (IgE) [21,22], thrombin [23], and total prostate-specific antigen (T-PSA) as a cancer marker [24]. Several research groups have explored the electrochemical detection of biological molecules with electrodes consisting of CNTs in their pristine or modified forms [19,20-27]. Since their introduction into electrochemistry, CNT electrodes have demonstrated enhanced sensitivity compared to conventional carbon electrode [6,20-24]. In addition, electronic changes in the behavior of SWCNTs have been detected when they interact with small biological molecules and proteins [14,19,23,24,27-29]. A field-effect transistor (FET), composed of an individual pristine SWCNT, changes resistance upon exposure to proteins [14].

It is important to develop convenient and inexpensive methods for detecting and quantifying multiple galectins in tissues, both for biological studies and for future diagnosis using clinical samples [4]. While the previous methods, such as ELISA and Western blotting, are useful in research, they may not be practical for the routine analysis of clinical samples, and most are limited to the detection of a single galectin [4]. Most current efforts to develop alternatives have been based on selective galectin labeling using chemical probes. In our experiments, we used SWCNTs as chemical probes. To our knowledge, this is the first time that D-(+)-galactose-conjugated SWCNTs have been used as chemical probes to detect galectin-3.

Based on our previous finding [30] that D-(+)-galactose at a concentration of $0.5-1 \mu \mathrm{g} / 100 \mu \mathrm{L}$ can bind to galectin-3 without structural changes, we investigated the binding affinity of galectin-3 at a nanoscale in electrochemical detection studies using a D-(+)-galactose-conjugated CNTs biosensor. Here, we determined the binding affinity of D-(+)-galactose-conjugated CNTs for the detection of the cancer marker galectin-3. This study is intended to provide preliminary information on the potential of D-(+)-galactose-conjugated CNTs as efficient nanobiosensors for the detection of the cancer marker galectin-3. 


\section{Results and Discussion}

\subsection{Binding Affinities of D-(+)-Galactose for Galectins}

From our preliminary studies of the binding of D-(+)-galactose to galectins at various concentrations $(0.25-2 \mu \mathrm{g} / 100 \mu \mathrm{L})$, we found that the absorbance intensity at $405 \mathrm{~nm}$ for the binding was approximately 0.5 for galectin- 3 and 0.9 for galectin- 8 . In addition, the fraction of galectins bound to D-(+)-galactose increased during up to $30 \mathrm{~min}$ of incubation time. Moreover, no structural damage to galectin-3 or galectin- 8 occurred in the binding studies, and the galectins retained their activity [30].

\subsection{Purification and Functionalization of SWCNTs}

As a first step in the biofunctionalization of SWCNTs, they were purified to remove amorphous carbon and transition metal impurities. In particular, the SWCNTs were purified to remove transition metals, such as $\mathrm{Fe}, \mathrm{Ni}$, and $\mathrm{Co}$, as these impurities may result in reduction-oxidation reactions during the manufacture of biosensors using electrochemical methods. After purification, a dispersion step was used to scatter the bundles of SWCNTs for the functionalization step.

The results of physical functionalization indicated that there was no $\pi$ stacking or mutual bonding forces between D-(+)-galactose and SWCNTs. However, the introduction of chemical functional groups, such as $-\mathrm{COOH}$ and $-\mathrm{COCl}$, did not cause any major structural alterations, as confirmed using field emission-scanning electron microscopy (FE-SEM; JEOL 6700F, JEOL, Tokyo, Japan), high-resolution transmission electron microscopy (HR-TEM; JEOL 2010F; JEOL), Fourier transform-Raman spectroscopy (FT-Raman; RM 1,000-Invia, Reinshaw, Gloucestershire, UK), and X-ray photoelectron spectroscopy (XPS; PHI 5100, Physical Electronics, Chanhassen, MN, USA). From the XPS $\mathrm{Cl}_{2 \mathrm{P}}$ analysis, no Cl-related peak was observed after treatment with $\mathrm{HNO}_{3} / \mathrm{NaClO}_{3}$, although a peak was observed after subsequent $\mathrm{SOCl}_{2}$ treatment. This latter observation confirmed the chemical functionalization on the surface of the SWCNTs. By contrast, significant structural alterations were observed in the FT-Raman analysis after using fluorination as another chemical functionalization process of SWCNTs. A coating of amorphous carbons on the surface of the fluorinated SWCNTs was observed using FE-SEM and HR-TEM, suggesting that excess fluorine bound to the surface of SWCNTs, which unzipped the SWCNT structure. This confirmed that fluorinated D-(+)-galactose-immobilized SWCNTs are unsuitable for use in the manufacture of electrochemical biosensors.

\subsection{Immobilization of D-(+)-Galactose on SWCNTS}

The FE-SEM and HR-TEM observations showed that the structure of the SWCNTs did not change after the reaction with D-(+)-galactose on SWCNTs functionalized with-COCl (Figure 1a and 1b). As shown in Figure 1c, the D-band related to $\mathrm{sp}^{3}$ was markedly increased due to $\mathrm{sp}^{3}$ bonding of D-(+)-galactose immobilized on the surface of the SWCNTs. After the reaction with D-(+)-galactose, the peak of $\mathrm{sp}^{3}$ bonding was increased markedly at $285.2 \mathrm{eV}$ in the XPS $\mathrm{C}_{1 \mathrm{~S}}$ analysis (Figure $1 \mathrm{~d}$ ). 
Figure 1. Images and analysis of SWCNTS after introducing D-(+)-galactose. (a) FE-SEM image, scale bar $100 \mathrm{~nm}$; (b) HR-TEM image, scale bar $0.5 \mu \mathrm{m}$; (c) D-band in FT-Raman; (d) XPS $\mathrm{C}_{1 \mathrm{~s}}$.
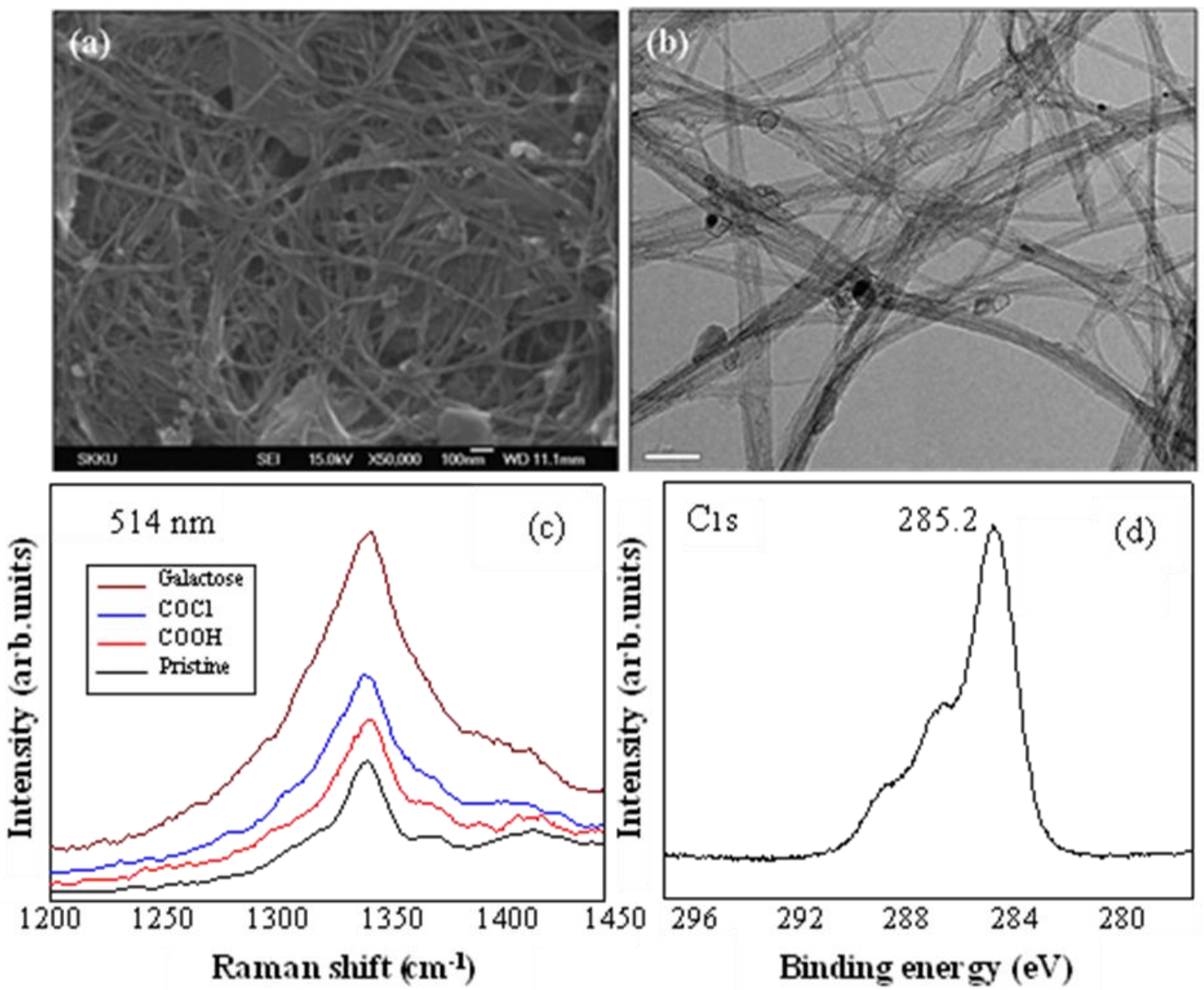

In addition, the hydroxyl group, hydrogen, and alcohol group of D-(+)-galactose that appeared after the introduction of D-(+)-galactose were observed using Fourier transform infrared spectroscopy (FT-IR; Bruker Optics, Billerica, MA, USA) (Figure 2). From these observations, we confirmed that the chemically functionalized SWCNTs were safely immobilized with D-(+)-galactose without undergoing any structural alterations. Finally, the SWCNTs immobilized with D-(+)-galactose were dispersed as individual SWCNTs in dichloroethane (DCE) and this observation was confirmed using atomic force microscopy (AFM; SPA-400, Seiko Instruments, Chiba-shi, Japan). 
Figure 2. FT-IR analysis after introducing D-(+)-galactose on the surface of SWCNT-COCl. The stretching frequencies of ether and alcoholic linkages (C-O-C,$-\mathrm{CH}_{2}-\mathrm{O}$ ) were seen around 1000 1300 $\mathrm{cm}^{-1}$, the carbonyl of carboxylic acid at $\sim 1700 \mathrm{~cm}^{-1}$, and the $-\mathrm{OH}$ of carboxylic acid at $\sim 2500$ and $\sim 3500 \mathrm{~cm}^{-1}$.

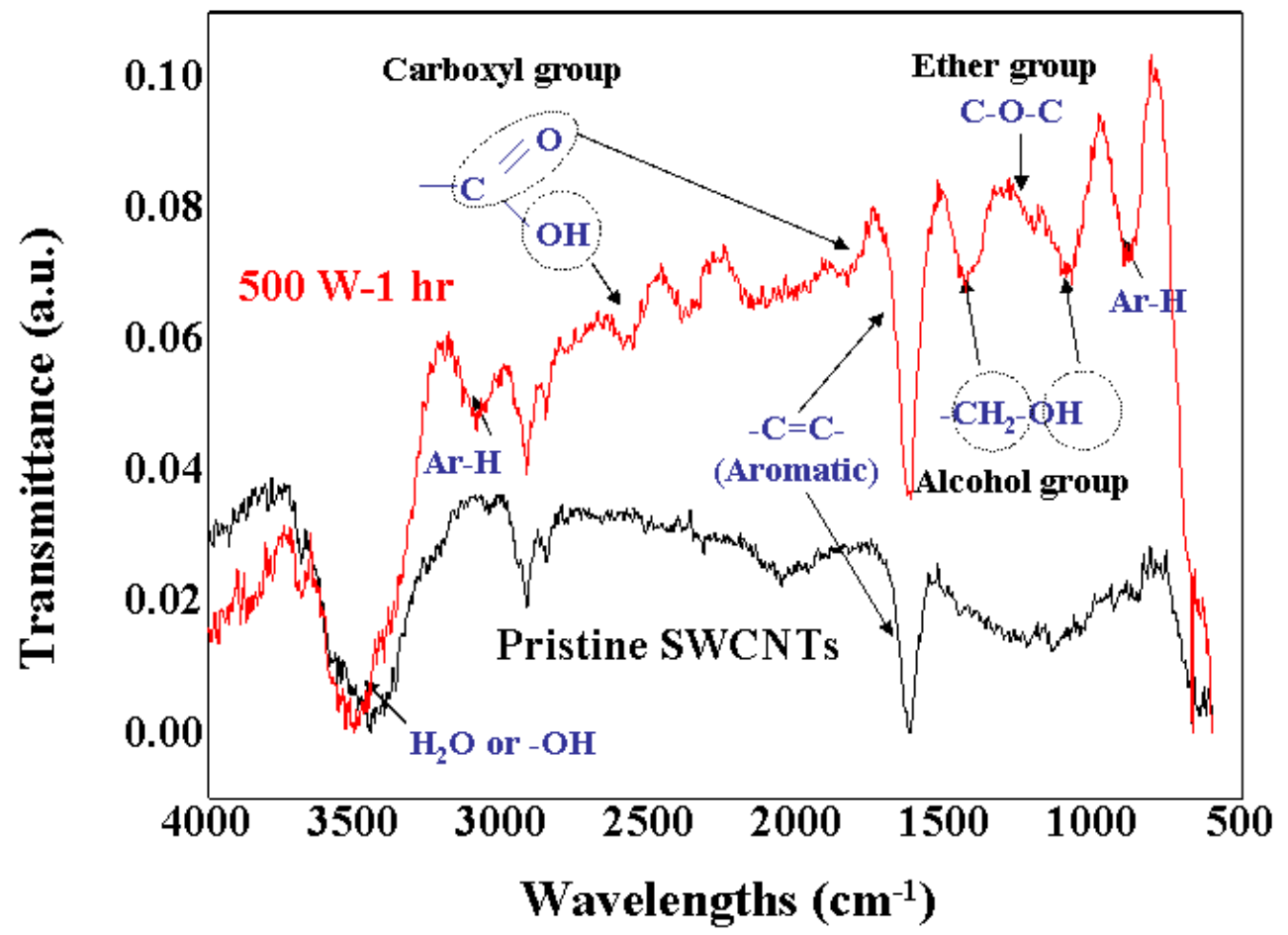

\subsection{Electrochemical Biosensor Using SWCNTs Immobilized with D-(+)-Galactose}

Individually dispersed SWCNTs were used to prepare electrochemical biosensors using D-(+)-galactose-immobilized SWCNTs. Well-dispersed SWCNTs in DCE were dropped onto the surface of a $\mathrm{SiO}_{2}$ substrate to fabricate molybdenum (Mo) electrodes as shown in Figure 3, and then used to measure current-voltage (I-V) characteristics and sensitivity based on differences in the resistance of samples in the presence or absence of galectin-3, without using other reference electrodes. Here, the primary effect of the binding of galectin-3 with SWCNTs is a charge-transfer reaction that involves the donation of electrons from galectin-3 to SWCNTs. The SWCNT-FET device may also be sufficiently sensitive to detect galectin-3 at low target concentrations. In these ways, the detection of galectin-3 is based not on measuring the oxidation-reduction potential of galectin-3 but on the I-V difference that occurs on the surface of SWCNTs when galectin-3 is bound to D-(+)-galactose-conjugated SWCNTs. Thus, despite a small electrochemical window, we used Mo electrodes without other reference electrodes to reduce dark current in SWCNT-FET and to increase the probability of p-type semiconduction during fabrication of an SWCNT-FET, and finally obtained high-sensitivity electrochemical biosensors. An electrochemical biosensor with a single strand of SWCNTs did not show sufficient sensitivity using Mo electrodes; it proved necessary to orient several dozen SWCNT strands between two Mo electrodes to function as a D-(+)-galactose-conjugated SWCNT biosensor. 
Figure 3. FE-SEM images of the molybdenum electrodes. (a) Twelve pairs of Mo Electrodes (inset: $1 \times 1 \mathrm{~cm}$ area of the patterned electrode); (b) Dispersed SWCNTs between two Mo electrodes in the region outlined with the black dotted circle in (a). The scale bars in (a) and (b) are $100 \mu \mathrm{m}$ and $0.5 \mu \mathrm{m}$, respectively.

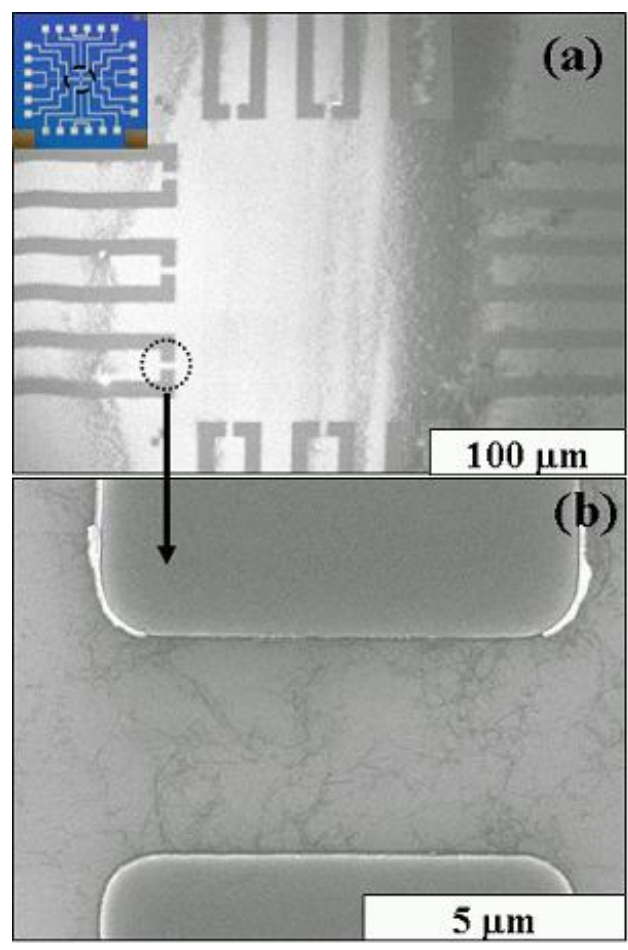

The sensitivity of a biosensor using SWCNTs dispersed between two Mo electrodes was defined as the difference in the resistance between sample solutions with or without galectin-3 and blank controls. The sensitivity was calculated using the formula $\left[\left(\mathrm{Rm}-\mathrm{R}_{0}\right) / \mathrm{R}_{0}\right]$, where $\mathrm{Rm}$ and $\mathrm{R}_{0}$ are the resistance of the samples with or without galectin-3 and that of the blank controls, respectively. Typical I-V measurements and sensitivities using the samples with or without galectin-3 are shown in Figure 4; the currents decreased after galectin-3 was bound to the D-(+)-galactose-conjugated SWCNTs based on the p-type characteristics of SWCNT-FETs [14,31]. Taking into account the fact that the SWCNTs exhibited p-type electronic behavior before adsorption of galectin-3, presumably due to doping from environmental oxygen, the current change after exposure to galectin-3 indicates an electron transfer from galectin-3 to the SWCNTs. A hybrid of galectin-3 and SWCNTs has been shown to be sensitive to the presence of D-(+)-galactose, which specifically binds to galectin-3, resulting in a decreased current. In this study, the sensitivity of the sample without galectin-3 was 18.7, whereas for samples with galectin-3, the maximum sensitivity was $31.3(0.3125 \mu \mathrm{g} / 100 \mu \mathrm{L})$. The sensitivity of the samples increased with the galectin-3 concentration over a limited concentration range (0.0156-0.03125 $\mu \mathrm{g} / 100 \mu \mathrm{L})$ (Figure 4b). From these results, we found that the resistance increased with the concentration of galectin-3 bound to the D-(+)-galactose-conjugated SWCNTs, and that the resistance was not further increased with concentration of galectin-3 higher than $0.03125 \mu \mathrm{g} / 100 \mu \mathrm{L}$ because of the saturation of the majority carriers on the SWCNTs by the action of galectin-3 as an electron donor [14,31]. Finally, we confirmed that the sensitivity for the samples with galectin-3 increased with the concentration of galectin-3 in the binding reaction over a limited concentration range. These observations imply that 
D-(+)-galactose-conjugated SWCNTs are potentially useful as electrochemical nanobiosensors for the cancer marker galectin-3.

Figure 4. Typical graphical data for (a) I-V measurements and (b) the sensitivities of samples with or without galectin-3. The typical I-V characteristics were measured using the prepared Mo electrodes. SWCNTs dispersed between the Mo electrodes were exposed to various concentrations of galectin-3 in PBS (two-fold serial dilutions from 0.5 to $0.0156 \mu \mathrm{g} / 100 \mu \mathrm{L}$ ). The results were obtained from three different experiments. Each bar represents the mean standard deviation.

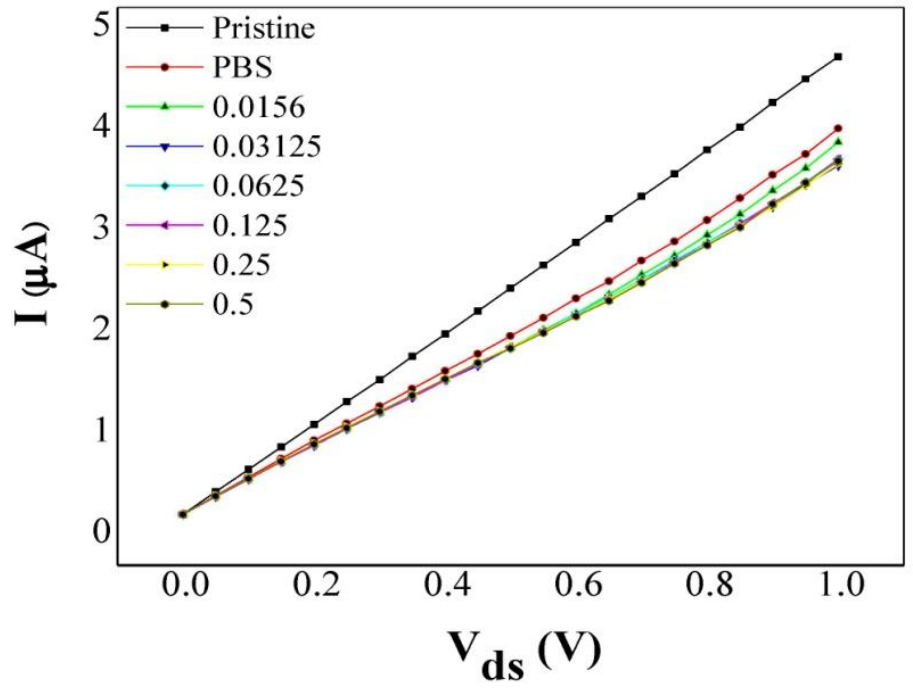

(a)

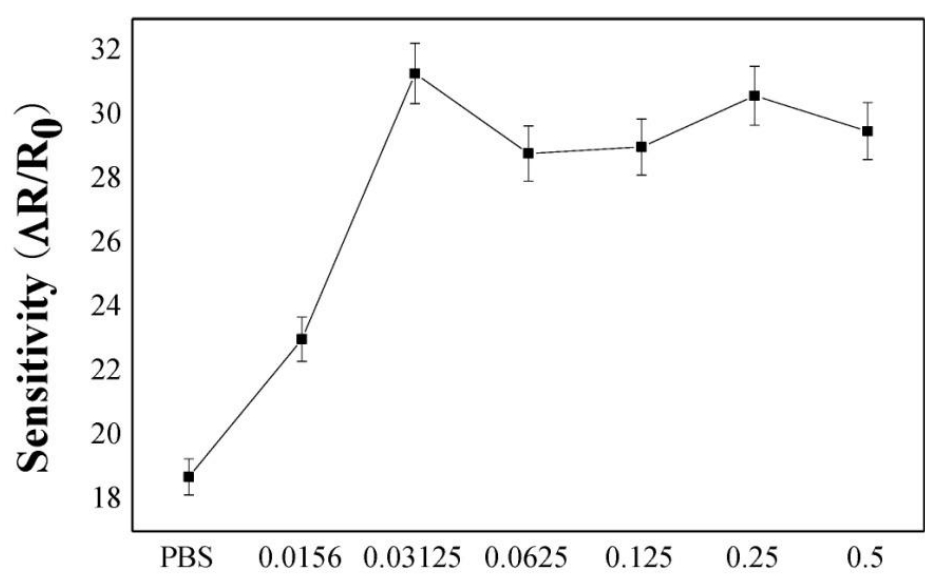

Amount of galectin-3 $(\mu \mathrm{g} / 100 \mu \mathrm{L})$

(b)

Galectin-3 is a non-immunoglobulin in nature and capable of specific recognition of and reversible binding to carbohydrate moieties of D-(+)-galactose-conjugated SWCNTs without altering the covalent structure of any of the recognized glycosyl ligands. In this study, SWCNT-FETs provided a simple detection mechanism between galectin-3 and D-(+)-galactose-conjugated SWCNTs without using reference electrodes. In addition, they resulted in a specific detection method with not only high selectivity based on the lectin characteristics of galectin-3 but also high 
sensitivity in the nanoscale range, based on a much larger diameter of galectin-3 compared with that of SWCNT channels (1-2 nm). Another advantage is that the binding between galectin-3 and D-(+)-galactose-conjugated SWCNTs is less reversible and more stable compared with non-covalent bonds in nature, such as antigen-antibody reactions based on non-covalent bonds including hydrogen bonds, electrostatic bonds, Van der Waals forces, and hydrophobic bonds. Therefore, the SWCNT-FET in this study offers several advantages, as mentioned above. Such an electrochemical sensor is small and rapid enough to detect the cancer marker galectin-3. In addition, the electrochemical sensor is extremely sensitive; all the current passes through the detection point. Most importantly, at a later stage, the SWCNTs-FET could be made specific to individual molecules; their response to different species could potentially be varied in a controlled way using chemical and biological functionalization.

In conclusion, this investigation of the binding sensitivity based on the resistance difference confirmed that D-(+)-galactose-conjugated SWCNTs are very efficient detection tools for selective binding to galectin-3, a biomarker on the surface of cancer cells. As the detection level demonstrated was within the nanoscale range $(0.0156-0.03125 \mu \mathrm{g} / 100 \mu \mathrm{L})$, the SWCNT conjugates developed in this study could be used as efficient nanobiosensors for galectin-3. Furthermore, a study of the binding sensitivity of D-(+)-galactose-conjugated SWCNTs for the cancer marker galectin-3 would provide comprehensive information pertinent to their potential for further development as nanobiosensors. To use SWCNT conjugates for cancer detection, it must be possible to measure galectin-3 reproducibly using a reliable and widely available assay. Further studies using SWCNT conjugates as new chemical probes are required to develop reliable methods for detecting galectin-3 in routine clinical samples.

\section{Experimental Section}

\subsection{Reagents}

Galectins were purchased from PeproTech Asia (Rehovot, Israel) and R \& D Systems (Minneapolis, MN, USA). Antibodies were purchased from Chemicon (Temecula, CA, USA) and Santa Cruz Biotechnology (Santa Cruz, CA, USA). $\beta$-D-(+)-Galactose and other chemical reagents were purchased from Sigma-Aldrich and used without further purification. Single-walled carbon nanotubes synthesized by the arc charge method were purchased from Iljin Nanotech (Seoul, Korea). All solutions were prepared using deionized water (Millipore, Bedford, MA, USA). The phosphate buffered saline (PBS) solutions adjusted to $\mathrm{pH} 7.4$ and $\mathrm{pH} 7.2$ as a supporting electrolyte were purchased from Invitrogen (Carlsbad, CA, USA).

\subsection{Instrumentation}

All experiments including purification, functionalization, immobilization, and dispersion steps, and electrochemical studies were performed using the research facilities at the Center for Nanotubes and Nanostructured Composites (CNNC) of Sungkyunkwan University (Suwon, Korea), including FE-SEM, HR-TEM, FT-Raman spectroscopy, XPS analysis, FT-IR, and AFM. 


\subsection{Preliminary Tests of Binding Affinity of D-(+)-Galactose and Galectin-3}

Galectin-3 solutions were prepared at initial concentrations of $0.25-2 \mu \mathrm{g} / 100 \mu \mathrm{L}$ in PBS (pH 7.4). All experiments were performed in the research facilities of the Biohealth Products Research Center (BPRC) of Inje University (Gimhae, Korea). In our preliminary test, we used ELISA, sodium dodecyl sulfate polyacrylamide gel electrophoresis (SDS-PAGE), and Bradford assays to examine the binding of galectins to D-(+)-galactose-conjugated carbon nanotubes [30].

\subsection{Purification and Functionalization of SWCNTs}

The SWCNTs including amorphous carbon particles as impurities were purified by heat treatment at $470{ }^{\circ} \mathrm{C}$ for $50 \mathrm{~min}$ under an air atmosphere. Then, transition metals used as catalysts in the process of SWCNT synthesis, such as $\mathrm{Fe}, \mathrm{Ni}$, and $\mathrm{Co}$, were removed to inhibit the oxidation and reduction reactions in the process of biosensor manufacturing by stirring in aqueous $\mathrm{HCl}$ for $24 \mathrm{~h}$.

Physical functionalization was performed by treating the surface of SWCNTs with D-(+)-galactose. SWCNTs were mixed with D-(+)-galactose at weight ratios of 1:30, 1:50, and 1:100. In addition, purified SWCNTs were prepared for chemical functionalization on their surfaces. Two different methods, i.e., introduction of $-\mathrm{COCl}$ groups at the end of the CNTs and fluorination of CNTs, were used for chemical functionalization of SWCNTs. For introduction of - $\mathrm{COCl}$ groups, aliquots of $50 \mathrm{mg}$ of purified SWCNTs were added to $100 \mathrm{~mL}$ of $70 \% \mathrm{HNO}_{3}$. Then, $250 \mathrm{mg}$ of $\mathrm{NaClO}_{3}$ was added to the resulting reactants under reflux at $100{ }^{\circ} \mathrm{C}$, followed by rinsing with distilled water and alcohol, and poured into $100 \mathrm{~mL}$ of DMF solution. After full dispersal, $50 \mathrm{~mL}$ of $\mathrm{SOCl}_{2}$ were added to the resulting reactants under reflux at $100{ }^{\circ} \mathrm{C}$. The introduction of $-\mathrm{COOH}$ and $-\mathrm{COCl}$ groups on the surface of SWCNTs was examined by FE-SEM, FT-Raman and XPS. For fluorination of CNTs, SWCNTs were introduced into the $\mathrm{Ni}$ reaction chamber and fluorinated by passage of 0.2 bar of $\mathrm{F}_{2}$ gas into the chamber at $25{ }^{\circ} \mathrm{C}$ for $30 \mathrm{~min}$. The fluorination of SWCNTs was also examined by HR-TEM, FE-SEM, and FT-Raman.

\subsection{Immobilization of D-(+)-Galactose on SWCNTs and Dispersion of SWCNTs Immobilized with} D-(+)-Galactose

An excess of D-(+)-galactose was added to the SWCNTs functionalized with $\mathrm{SOCl}_{2}$. The reactants were subsequently kept at $280{ }^{\circ} \mathrm{C}$ under a nitrogen atmosphere for $12 \mathrm{~h}$, and rinsed with distilled water and alcohols. The immobilization was observed using FE-SEM, HR-TEM, FT-Raman, XPS, and FT-IR. The SWCNTs immobilized with D-(+)-galactose were dispersed in DCE using ultrasonic treatment and centrifuged at 17,000 rpm for $30 \mathrm{~min}$. The supernatant was separated and spin-coated on the surface of silicon substrates. The degree of dispersion was observed using AFM.

\subsection{Biosensor Preparation and Electrochemical Analysis}

The samples of $0.1 \mathrm{mg}$ of SWCNTs immobilized with D-(+)-galactose were sonicated in $100 \mathrm{~mL}$ of DCE for $15 \mathrm{~h}$. After centrifuging the sample at 27,460 g for $3.5 \mathrm{~h}$, the supernatant was dropped on a prepatterned $\mathrm{SiO}_{2}$ substrate. The Mo electrode pattern was printed on the $\mathrm{SiO}_{2}$ substrate using conventional photolithography. Well-dispersed SWCNTs in DCE were dropped onto the patterned Mo 
electrodes. Each electrode sample with well-dispersed SWCNTs was dried in air overnight. The morphology of the dispersed SWCNTs between the two Mo electrodes was observed using FE-SEM. The density of SWCNTs was determined by controlling the amounts of CNTs in the solution. The typical I-V characteristics were measured using the prepared Mo electrodes. In order to measure the sensitivity of the biosensor, the SWCNTs dispersed between the Mo electrodes were exposed to various concentrations of galectin-3 in PBS (two-fold serial dilutions from 0.5 to $0.0156 \mu \mathrm{g} / 100 \mu \mathrm{L}$ ). The change in resistance was measured for samples with or without galectin-3. Controls were also measured using blank tests.

\section{Conclusions}

The binding affinities of D-(+)-galactose-conjugated SWCNTs for galectin-3 were determined using the electrochemical detection of resistance differences based on typical I-V characterization. Samples with galectin-3 showed greater sensitivity than those without galectin-3. In addition, the sensitivity of the samples with galectin-3 increased with the amount of galectin-3 in the binding reaction with the components at acceptable levels. The biofunctionally conjugated SWCNT showed electrochemical sensing levels in the nanoscale range for detecting the cancer marker galectin-3. Therefore, D-(+)-galactose-conjugated SWCNTs are potentially useful electrochemical nanobiosensors for the cancer marker galectin-3.

\section{Acknowledgements}

This work was supported by Inje-University Research Grant in 2010.

\section{References}

1. Lis, H.; Sharon, N. Protein glycosylation: Structural and functional aspects. Eur. J. Biochem. 1993, 218, 1-27.

2. Leffler, H. Galectins structure and function-A synopsis. Results Probl. Cell Differ. 2001, 33, 57-83.

3. Califice, S.; Castronovo, V.; van den Brûle, F. Galectin-3 and cancer (Review). Int. J. Oncol. 2004, 25, 983-992.

4. Pieters, R.J. Inhibition and detection of galectins. ChemBioChem 2006, 7, 721-728.

5. Wang, J. Carbon-nanotube based electrochemical biosensors: A review. Electroanalysis 2005, 17, $7-14$.

6. Britto, P.J.; Santhaman, S.K.V.; Ajayan, P.M. Carbon nanotube electrode for oxidation of dopamine. Bioelectrochem. Bioenerg. 1996, 41, 121-125.

7. Guiseppi-Elie, A.; Lei, C.; Baughman, P.H. Direct electron transfer of glucose oxidation on carbon nanotubes. Nanotechnology 2002, 13, 559-564.

8. Li, J.; Ng, H.T.; Cassell A.; Fan, W.; Chen, H.; Ye, Q.; Koehne, J.; Han, J.; Meyyappan, M. Carbon nanotube nanoelectrode array for ultrasensitive DNA detection. Nano Lett. 2003, 3, 597-602. 
9. Goodung, J.J.; Wibowo, R.; Liu, J.; Yang, W.; Losic, D.; Orbons, S.; Mearns, F.J.; Shapter, J.G.; Hibbert, D.B. Protein electrochemistry using aligned carbon nanotube arrays. J. Am. Chem. Soc. 2003, 125, 9006-9007.

10. Lin, Y.; Lu, F.; Tu, Y.; Ren, Z. Glucose biosensor based on carbon nanotube nanoelectrode ensembles. Nano Lett. 2004, 4, 191-195.

11. Soundarrajan, P.; Patil, A.; Dai, L. Surface modification of aligned carbon nanotube arrays for electrochemical sensing applications. J. Vac. Sci. Technol. 2003, 21, 1198-1201.

12. Gao, M.; Dai, L.; Wallace, G.G. Biosensors based on aligned carbon nanotubes coated with inherently conducting polymers. Electroanalysis 2003, 15, 1089-1094.

13. Wang, J.; Musameh, M. Carbon nanotube/teflon composite electrochemical sensors and biosensors. Anal. Chem. 2003, 75, 2075-2079.

14. Star, A.; Gabriel, J.C.P.; Bradley, K.; Gruner, G. Electronic detection of specific protein binding using nanotube FET devices. Nano Lett. 2003, 3, 459-464.

15. Luong, J.H.; Hrapovic, S.; Wang, D.; Bensebaa, F.; Simard, B. Solubilization of multiwall carbon nanotubes by 3-aminopropyltriethoxysilane toward the fabrication of electrochemical biosensors with promoted electron transfer. Electroanalysis 2004, 16, 132-139.

16. Wang, J.; Liu, G.; Jan, M.R. Ultrasensitive electrical biosensing of proteins and DNA: Carbon-nanotube derived amplification of the recognition and transduction events. J. Am. Chem. Soc. 2004, 126, 3010-3011.

17. Trojanowicz, M.; Mulchandani, A.; Mascini, M. Carbon nanotubes-modified screen-printed electrodes for chemical sensors and biosensors. Anal. Lett. 2004, 37, 3185-3204.

18. Okuno, J.; Maehashi, K.; Matsumoto, K.; Kerman, K.; Takamura, Y.; Tamiya, E. Single-walled carbon nanotube-arrayed microelectrode chip for electrochemical analysis. Electrochem. Commun. 2007, 9, 13-18.

19. Besteman, K.; Lee, J.O.; Wiertz, F.G.M.; Heering, H.A.; Dekker, C. Enzyme-coated carbon nanotubes as single-molecule biosensors. Nano Lett. 2003, 3, 727-730.

20. Kim, J.P.; Lee, B.Y.; Hong, S.; Sim, S.J. Ultrasensitive carbon nanotube-based biosensors using antibody-binding fragments. Anal. Biochem. 2008, 381, 193-198.

21. Maehashi, K.; Katsura, T.; Kerman, K.; Takamura, Y.; Matsumoto, K.; Tamiya, E. Label-free protein biosensors based on aptamer-modified carbon nanotube field-effect transistors. Anal. Chem. 2007, 79, 782-787.

22. Maehashi, K.; Matsumoto, K.; Takamura, Y.; Tamiya, E. Aptamer-based label-free immunosensors using carbon nanotube field-effect transistors. Electroanalysis 2009, 11, 1285-1290.

23. So, H.; Won, K.; Kim, Y.H.; Kim, B.; Ryu, B.H.; Na, P.S.; Kim, H.; Lee, J. Single-walled carbon nanotube biosensors using aptamers as molecular recognition elements. J. Am. Chem. Soc. 2005, 127, 11906-11907.

24. Okuno, J.; Maehashi, K.; Kerman, K.; Takamura, Y.; Matsumoto, K.; Tamiya, E. Label-free immunosensor for prostate-specific antigen based on single-walled carbon nanotube array-modified microelectrodes. Biosens. Bioelectron. 2007, 22, 2377-2381.

25. Liu, G.; Riechers, S.L.; Mellen, M.C.; Lin, Y. Sensitive electrochemical detection of enzymatically generated thiocholine at carbon nanotube modified glassy carbon electrode. Electrochem. Commun. 2005, 7, 1163-1169. 
26. Lawrence, N.S.; Wang, J. Chemical adsorption of phenothiazine dyes onto carbon nanotubes: Toward the low potential detection of NADH. Electrochem. Commun. 2006, 8, 71-76.

27. Zhao, Q; Gan, Z.; Zhuang, Q. Electrochemical sensors based on carbon nanotubes. Electroanalysis 2002, 14, 1609-1613.

28. Bradley, K.; Briman, M.; Star, A; Gruner, G. Charge transfer from adsorbed proteins. Nano Lett. 2004, 4, 253-256.

29. Boussaad, S.; Tao, N.J.; Zhang, R.; Hopson, T.; Nagahara, L.A. In situ detection of Cytochrome C adsorption with single walled carbon nanotube device. Chem. Commun. 2003, 13, 1502-1503.

30. Park, Y.K.; Bold, B.; Cui, B.C.; Bai, J.Q.; Lee, W.K.; Shim, Y.K. Binding affinities of carbohydrate-conjugated chlorins for galectin-3. Bull. Kor. Chem. Soc. 2008, 29, 130-134.

31. Kong, J.; Franklin N.R.; Zhou, C.; Chapline, M.G.; Peng, S.; Cho, K.; Dai, H. Nanotube molecular wires as chemical sensors. Science 2000, 287, 622-625.

(C) 2011 by the authors; licensee MDPI, Basel, Switzerland. This article is an open access article distributed under the terms and conditions of the Creative Commons Attribution license (http://creativecommons.org/licenses/by/3.0/). 